\title{
ROTATIONAL PATH PARTIAL DENTURES: AN UNDERUTILIZED TREATMENT MODALITY IN AESTHETIC DENTALMEDICINE
}

\author{
Adam Perry Tow ${ }^{1 a^{*}(i)}$ \\ 'Department of Restorative Dental Sciences, College of Dentistry, University of Florida, Gainesville, FL 32610, USA
}

aMBA, DMD Candidate

\begin{abstract}
DOI: https://doi.org/10.25241/stomaeduj.2018.5(4).art.7
\end{abstract}
Aim: To design a highly aesthetic prosthesis at low cost, which replaces maxillary anterior teeth without showing removable denture clasps.

Summary: Today's clinical practice is highly dictated by the increasingly demanding aesthetic standards of the modern patient. While advances in biomaterials and titanium osseo-integrated implants have made replacing missing teeth possible in a natural-looking way, many patients are not candidates for these fixed restorations due to physiological or financial barriers. In this case report, a patient with a history of anterior maxillary incisor partial-edentulism for whom fixed restorations were not feasible was treated using a rotational path of insertion partial denture.

With this technique, the author was able to design a removable partial denture with no clasps showing, irrespective of the smile line height.

The final result completely obscures the retentive mechanisms upon smiling and is highly aesthetic, on par with implant-retained fixed restoration, at a fraction of the cost and without the associated risks and complications of surgically-driven prosthetic cases.

Learning Points: This article will review this case and the supporting literature, as well as provide guidance on laboratory prescription writing and optimal case selection.

Keywords: Denture, Partial, Removable (D003832) Esthetics, Dental (D004955) denture, partial, removable (D003832) esthetics, dental (D004955).

\begin{tabular}{|c|}
\hline $\begin{array}{l}\text { OPEN ACCESS This is an Open Access } \\
\text { article under the CC BY-NC } 4.0 \text { license. }\end{array}$ \\
\hline$E_{\text {Peer-Reviewed Article }}$ \\
\hline $\begin{array}{l}\text { Citation: Tow AP. Rotational path partial dentures: } \\
\text { An underutilized treatment modality in aesthetic } \\
\text { dental medicine. Stoma Edu J. 2018;5(4):263-269 }\end{array}$ \\
\hline $\begin{array}{l}\text { Academic Editor: Alexandru Eugen Petre, DDS, } \\
\text { PhD, Professor, "Carol Davila"University of Medicine and } \\
\text { Pharmacy, Bucharest, Romania }\end{array}$ \\
\hline $\begin{array}{l}\text { Received: December 05, } 2018 \\
\text { Revised: December 07, } 2018 \\
\text { Accepted: December 14, } 2018 \\
\text { Published: December 15, } 2018\end{array}$ \\
\hline $\begin{array}{l}\text { *Corresponding author: } \\
\text { Dr. Adam P. Tow, MBA } \\
\text { Department of Restorative Dental Sciences, College } \\
\text { of Dentistry, University of Florida, } 1395 \text { Center Dr, } \\
\text { Gainesville, FL 32610, USA } \\
\text { Tel/Fax: + 1 1970-823-2605, } \\
\text { e-mail: aptow@dental.ufl.edu }\end{array}$ \\
\hline $\begin{array}{l}\text { Copyright: } \odot 2018 \text { the Editorial Council for the } \\
\text { Stomatology Edu Journal. }\end{array}$ \\
\hline
\end{tabular}

\section{Introduction and Background}

Today's clinical practice is highly dictated by the increasingly demanding aesthetic standards of the modern patient. While advances in biomaterials and titanium osseo-integrated implants have made replacing missing teeth possible in a natural-looking way, many patients are not candidates for these fixed restorations due to physiological or financial barriers. In this case report, a patient with a history of anterior maxillary incisor partial-edentulism for whom fixed restorations were not feasible was treated using a rotational path of insertion partial denture.

Removable partial dentures, abbreviated RPDs, are a popular, inexpensive treatment option for the partially dentate patient seeking full arch rehabilitation. Generally, well-designed RPDs are composed of the following basic components: (1) a cast metal major connector that forms the majority of the body of the prosthesis, (2) areas of metallic mesh upon which acrylic gingiva and denture teeth are affixed, (3) guide planes, metal areas which contact the, often purposely adjusted, proximal teeth in edentulous areas, (4) cast metal occlusal rests which fit into messio-occlusal rest seats prepped into the teeth, (5) and metal retentive and reciprocal clasp elements which engage undercuts on the buccal (or lingual) surface, which are connected to the prosthesis via connecting metal referred to as the minor connector.
The clasping systems which attach the prosthesis to the dentition are generally cast metal, though soldered wrought wire, and thermoplastic options do exist $[1,2]$. Indeed, various components of the RPD may also be made with thermoplastic elements, though in cases similar to the one reviewed here, the author will suggest that the rotational path RPD design is clinically superior.

The rotational path of insertion removable partial denture was first reported on by Humphereys in 1935 and credited to Hallen Back [3]. The concept is broadly divided into two categories, Category I and Category II prosthetics $[4,5]$. The former are useful when mesially tipped molars do not have adequate buccal undercuts for traditional clasp retention. The latter are the subject of this article and are useful in Kennedy Class IV and similar situations where missing anterior teeth need to be replaced esthetically. The advantage of the type of prosthetic discussed herein is that it has no anterior clasps, making the transition from prosthesis to natural tooth seamless.

Because the design of these prosthesis is considered "complicated" by many practitioners, this technique is seldom taught in the dental school curriculum. According to Jacobson, et. al., nearly $20 \%$ of surveyed prosthodontists report only a "superficial understanding" of rotational path RPDs [5]. The author believes this lack of familiarity is likely only increased as our profession has turned its focus 


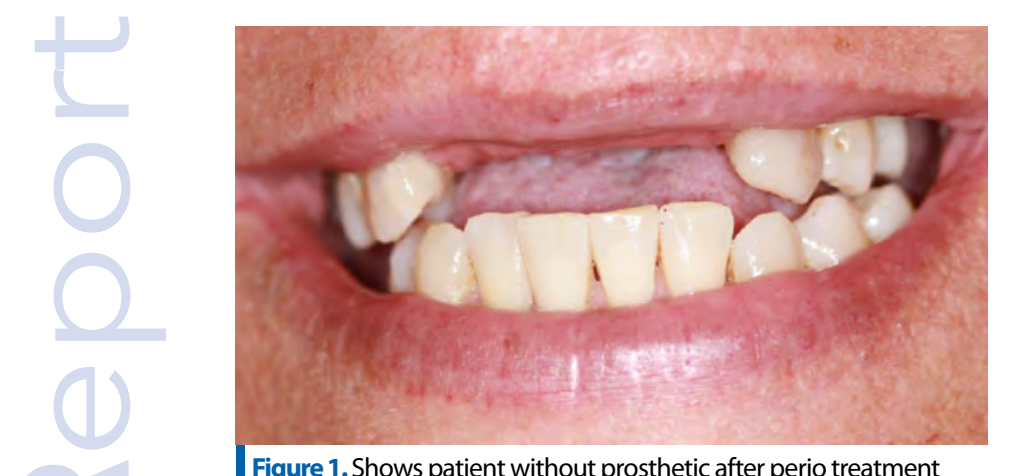

Figure 1. Shows patient without prosthetic after perio treatment

toward osseointegrated implants, new restorative materials, and fixed treatment modalities.

As will be shown here, this treatment modality is both accessible and attractive to patient and clinician alike. While the rotational path RPD may lack the 'glamor' of newer surgical fixed treatments, it more than compensates with highly aesthetic treatment results which outshine other removable options and even rival more complex treatments.

\section{Case Presentation}

\subsection{Initial Presentation and Phase 0-II Therapy}

A forty-year old female patient presented to the clinic with a history of maxillofacial trauma, having lost her maxillaryincisorsinanequestrianaccidentinheryouth. She presented to the clinic with an ill-fitting flipper with which she was unhappy. Her chief complaint was her smile aesthetics and poor functioning prosthetic, as well as some acute pain on tooth \#18. Her medical history was generally non-contributory to her dental evaluation, except that she was a 20 pack-year smoker who quit smoking over the course of her dental treatment described here. She also disclosed that her smile had negative effects on her self-esteem and discussed the significance of her "semi-colon" wrist tattoo with her dental provider. (Semi-colon tattoos are a symbol of the suicide struggle awareness and prevention movement.) As might be expected, data have shown that comorbid depression and anxiety are associated with partial edentulism [6].

In addition to the maxillary partial endentualism, she also presented with need for acute treatment of a mandibular molar which was extracted due to carious invasion of the pulp. The patient examination classified her as high caries risk, with several active lesions and missing teeth on both arches. The patient was diagnosed with mild-moderate chronic generalized periodontal disease with localized moderate-severe disease around the upper right first molar. Phase I treatment evaluation showed substantial improvement in periodontal health after scaling and root planning therapy, including stabilization and marked improvement of the periodontal health of the maxillary molar. Fig. 1 shows a photograph of the patient after completion of initial periodontal treatment. Caries risk was also

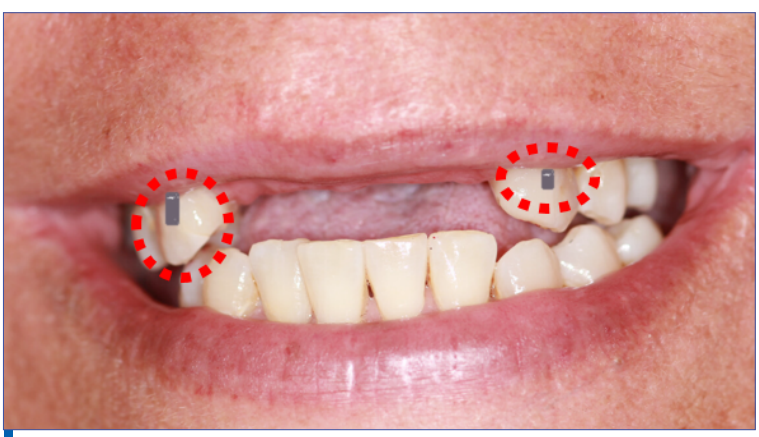

Figure 2. Shows a simulated digital smile analysis, revealing that i-bar clasps would likely create an unattractive metal display.

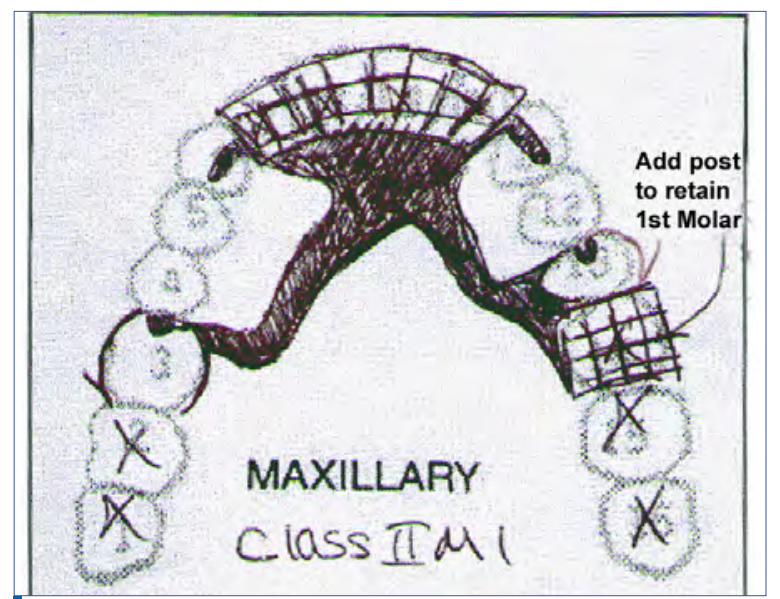

Figure 3. The figure shows the drawn lab script for rRPD.

decreased by extensive restorative dentistry and significant improvement in patient home-care via oral hygiene instructions delivered in the dental operatory.

\subsection{Definitive Treatment Planning and Phase III Therapy}

With active dental disease controlled, the patient was cleared for definitive prosthetic treatment. Because finances were a significant factor in treatment, implant or traditional fixed bridge therapy were not considered. This made removable partial dentures the only financially and medically viable treatment available. Initially, a classic metal-acrylic RPD design was proposed, with infrabulge or i-bar style clasps utilized on the maxillary canine teeth. A similar design was proposed for the lower arch. Smile analysis, however, as simulated in Fig. 2 showed that this would likely cause the maxillary anterior clasps to be visible upon smiling and functional movement. Though infrabulge clasps are a good first instinct for the RPD architect attempting to obscure clasps, they are often contraindicated in the aesthetically conscious young patient whose labial tissues and gingival show cause the clasps to be visible, especially in the anterior maxillary arch [7]. An experienced dentist can quickly ascertain whether clasps will be visible by visual inspection; however, digital prosthetic smile design techniques such as the one show in Fig. 2 are simple and fast ways to communicate the 

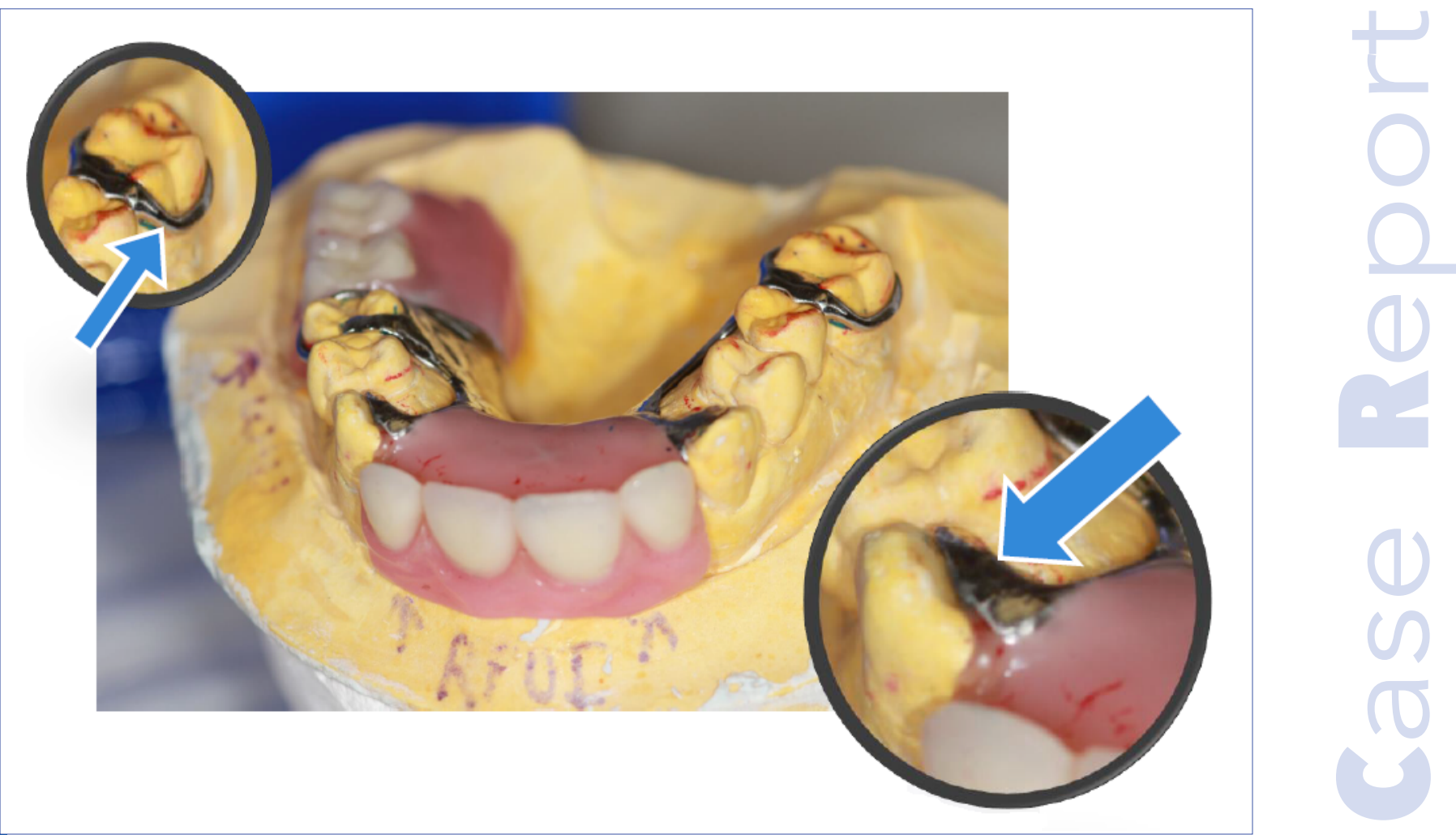

Figure 4. Shows the prosthetic on the master cast, with blue arrows indicating the conventional posterior clasp (left) and the long anterior rest seats (right).

issue to a patient, if needed. Several options exist to address this issue of exposed clasps and they will be discussed now in brief. One option is to use a Valplast or similar thermoplastic clasp which can be tooth colored. This option has several limitations. First, the clasp is still visible. It is merely less noticeable. Second, thermoplastic materials are more prone to fatigue and fracture [1,2]. Though most clinical studies support the use of thermoplastics for their aesthetic advantages, their reported "clinical acceptability" does not make them equals with respect to mechanics to their metal counterparts. A second option is the use of precision attachments. These come in two varieties, intra and extracoronal attachment systems [7]. The intracoronal variety have several limitations including that they require a certain level of laboratory sophistication, i.e. the precision aspect of the attachment requires the parts mate exactly, without the inherent leeway about a broad tooth contour traditional bulge-articulating clasps have. Additionally, these clasps require more significant preparation of the teeth, and cementation, both of which introduce obvious short and long-term clinical issues for both clinician and patient. The second subtype of precision attachment are extracornonal attachments, which function by cantilevering a hoop off of the abutment teeth into which a pin on the RPD fits. This requires a certain level of space within the prosthesis and presents with issues of retaining the attachment, as well as the introduction of a fixed, iatrogenic plaque trapping area beneath the attachment. Though not an issue in this case, these attachment systems may also be contraindicated in the elderly or other patients with limited dexterity. Given the large comorbidity associated with low dexterity and partial edentualism, this is a significant limitation of these systems. As a history of high caries risk and significant periodontal disease virtually ubiquitous among partially edentulous patients, there is ample reason for caution in prescribing these treatment options.

\subsection{Rotational Path Partial Denture Treatment Option}

The rotational path of insertion removable partial denture (rRPD) was selected as the treatment modality for this case in the maxillary arch, as shown in the lab prescription reproduced in Fig. 3. Due to the available mandibular premolar abutment teeth, a traditional i-bar design was possible for the lower arch. Therefore, the discussion that follows will concentrate on the maxilla were the rRPD was used.

The rRPD was first introduced in the 1930's by Hallen Back and has been investigated by several more contemporary authors, most notably Krol and Jacobson $[4,5,8]$. Unfortunately, the technique is virtually never taught in the predoctoral dental curriculum and it was reported by Jacobson in 1994 that nearly $20 \%$ of surveyed prosthodontist felt they had only a "superficial" understanding of the topic [4]. With the recent focus of our profession on osseointegrated fixed restorations, it is doubtful that this number has decreased in recent years. That said, especially with the risks associated with the increasingly popular bisphosphonate therapy and several other financial and medical issues which 


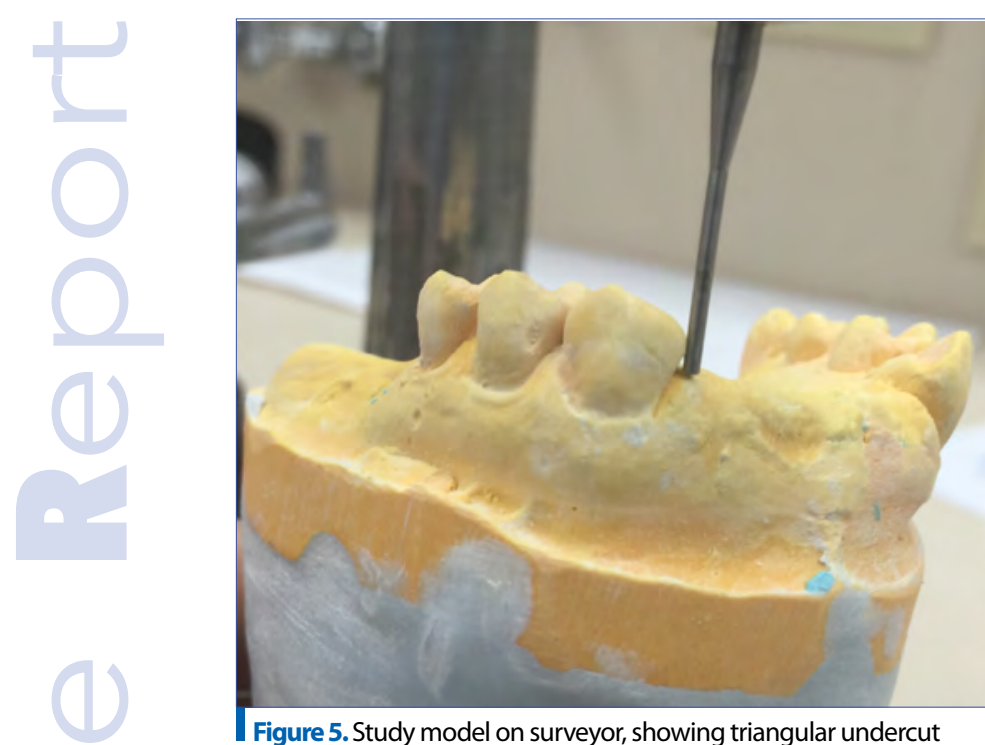

needed for rigid retainer.

complicate or contraindicate implants, the need for aesthetic removable options for patients is certainly real. The rRPD relevant to our discussion is the Category II Rotational Path of Insertion RPD. Because of its usefulness in aesthetic cases and its accessibility, it is hoped that adding a representative case study to the literature will contribute positively toward informing clinicians about this treatment option, and dispelling the notion that it is "too complicated" for the average dental practitioner or lab to be viable in modern practice.

\subsubsection{Review of rRPD Case Selection and Basic Components}

The rRPD is a fully cast framework RPD which employs a specialized guide plane called a rigid retainer to lock into mesial undercuts and a curvilinear path of insertion to retain a denture with no anterior clasps. The rRPD has the following basic components: (1) anterior mesial undercuts (2) rigid retainers (3) long rest seats on the claspless abutment teeth (4) conventional posterior clasps. Several of these features can be appreciated by examining Fig. 4, which shows the prosthetic on the master cast. Because of the non-linear path of insertion, Kennedy modification spaces complicate the treatment. To accommodate these, the clinician must carefully assess rotational path during guide plane enameloplasty planning. There are paralleling devices which can be used to accomplish this; however, it is suggested that, especially for the occasional prescriber, these cases which require complex guide planes on modification space teeth be avoided. Instead, the ideal case for this treatment is a Kennedy Class IV or Class I or II with a single anterior modification space. In these cases, the surveying can be done with relatively little additional knowledge or skill, and rRPD specific challenges with cast framework try-in are minimized. Of course, there are many other cases in which rRPDs

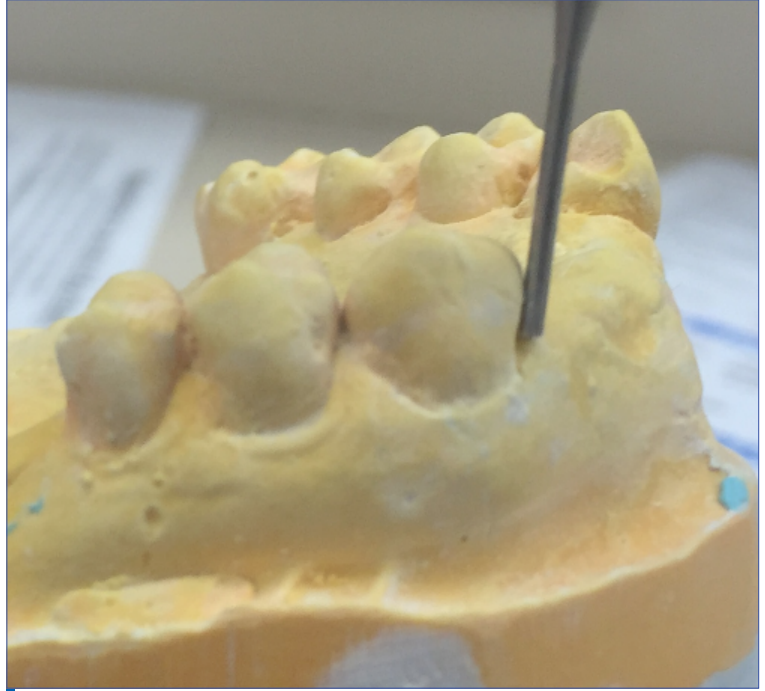

Figure 6. Shows the analyzing rod parallel to the mesial undercut with the terminus at the'A-point' of rotational path.

are useful, such as so-called Category I rRPDs which can be used for tipped posterior molars [5]. However, the goal of this paper is to demonstrate what the author believes is the simplest and most powerful use of the technique, the anterior edentualism situation. This case is a prototypical example of a straightforward case for which rRPD is indicated: Infrabulge or thermoplastic clasps are aesthetically untenable, and there is a single anterior edentulous space continuous with distant posterior tooth abutments.

\subsubsection{Surveying Concepts}

To design the prosthetic, study models were analyzed with the occlusal plane roughly parallel to the base of the surveyor. The analyzing rod is placed on the edentulous anterior ridge on the mesial surfaces of both abutment teeth, ideally canines. A significant undercut should be identified or created by enameloplasty or addition of resin-based composite, the latter being the case here where the undercuts were augmented with composite. A triangular wedge of light should shine through the space between the rod, tooth, and gingival stone. The occlusal plane and tilted surveying are shown in Figs. 5 and 6 , respectively. The cast is now tilted so that the analyzing rod contacts the full incisocervical mesial surface of the abutments. In other words, the "triangle" is now closed, with the analyzing rod placed on its hypotenuse, that is, flush to the mesial surface, as shown in Fig. 6 . The terminus of the analyzing rod is now touching the gingiva at the "A-point," the pivot around which the rotational path of insertion will rotate in the final prosthetic [4]. The posterior teeth can now be surveyed for facial undercuts on the posterior abutment teeth which will utilize standard cast circumferential or CC-clasps. The author recommends a lingual arm for counter retention of the posterior clasp to reduce the complexity of the metal framework and better accommodate adjustment, if needed. 


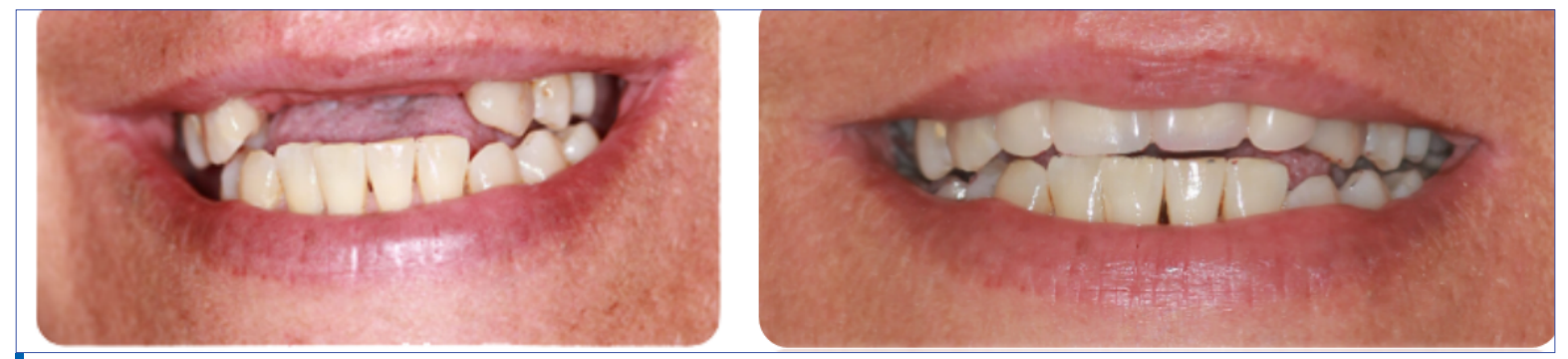

Figure 7. Are pre and post treatment photographs of the patient at rest showing the highly aesthetic results.

\subsubsection{Biomechanics}

The prosthetic works based on a simple principle common to all RPDs: that vertical displacing forces perpendicular to the surveyed occlusal plane must be resisted to keep the prosthetic engaged and prevent dislodgment during function. In the posterior, this is accomplished by a traditional CC-clasp, wherein the buccal contour of the clasped tooth entraps the clasp and secures it against a physiological vertical dislodging force. The anterior portion of the prosthetic relies on the 'rigid retainer' which is entrapped by the 'triangular' mesial undercut, thereby also resisting the vertical force, as the framework is 'wedged under' the tooth. For the rRPD, only a rotationally directed force about the radius defined by the A-point at the mesiogingival terminus of the mesial abutment teeth can dislodge (or seat) the prosthetic. Visualizing how the prosthetic would 'get stuck' by the posterior buccal buldge and anterior mesial undercuts will help the clinician appreciate the simple principle by which these prosthetics operate.

\subsection{Teeth Preparations}

As discussed, the rigid retainer abutting teeth may require some adjustment to accommodate the prosthesis; however, the natural anatomy of canines often lends itself to this application unmodified. The rigid retainer teeth should be supported by long anterior rests which extend from the mesial surface approximately half the mesio-distal distance of the tooth. The remainder of the rest seats are prepared traditionally.

\subsection{Fabrication of Prosthesis}

After preparation and impression, the metal framework is fabricated and the patient is reappointed for try-in. At this appointment, the doctor should verify the fit of the prosthesis, and ensure the partial framework fits without any occlusal interference. It is recommended that patient education regarding the insertion of the prosthesis begin at this point, so the wax try-in and delivery appointments are of increasing instructional value. Wax rims should be fabricated and tested after confirming the metalonly framework is satisfactory. Teeth should be set, either on-site, or by an off-site lab technician and the processing and delivery of the final rRPD should proceed as in any other RPD case.

\subsubsection{Delivery \& Patient Education}

Since the rRPD should not be inserted or removed along a straight-line path, it is important to educate the patient in the importance of maintaining the contour of the abutment teeth by ensuring that removal of the prosthesis does not grind the anterior abutment teeth. In other words, and especially in the cases of milder anterior mesial undercuts, the patient must always remove the prosthetic along the arc path of insertion to avoid wearing down the undercuts. Additionally, it is vital that the patient is informed that $s$ /he must inform other providers (e.g. hygiene) that the prosthesis is an rRPD, and ideally remove it him/herself. In this case, the patient was extremely pleased with the results which are shown in Fig. 7. She was able to achieve a very natural looking smile that provided her the desired form and function and increased her self-confidence. Due to the financial constraints, a result at this level of esthetics would have been all but impossible without the rRPD concept. It is fair to say that without using a rotational path, the treatment would have fallen short on its most fundamental goal to restore not only the biomechanical but the psychosocial function of the dentition.

\subsection{Additional Benefits and Considerations}

Some authors have also suggested that the claspless and flush anterior design promotes better periodontal health [9]. Given the association of partial edentualism and poor periodontal health, this is a noteworthy consideration.

Here are clear psychological benefits of the claspless appearance of the rRPD, both in how it looks and in that it is "different" from a traditional RPD.

In this case, the clinician made the decision to add a pin to retain the distal molar which was also replaced by the maxillary prosthesis. It is possible to add such retention features to the entirety of the anterior segment. This is particularly useful in cases where the residual ridge is more posteriorly located relative to the mandible. This will provide additional reinforcement where the denture teeth are retained by very thin acrylic embedded in the framework only. However, while pins are encouraged if needed, "bead retention" is discouraged by lab technicians as it may complicate the fabrication process and provides relatively little benefit [10]. 


\section{Learning Summary Points}

The case presented here should serve as a prototype for both the clinical decision tree that should be used to evaluate the potential rRPD case, as well as the steps to designing the prosthesis. The following learning points should help guide the clinician.

1. A highly aesthetic, claspless RPD can be designed easily and quickly, yielding a removable prosthesis that rivals its fixed counterparts and is a sound financial proposition.

2. The ideal case is a Kennedy Class IV or similar situation of a single space of anterior edentulism, such as Class I or II with only one modification space.

3. Smile analysis should have precluded the use of infrabulge clasps or fixed solutions.

4. Standard equipment for RPD design can be used with little additional knowledge, namely a standard surveyor with analyzing rod. The lack of familiarity with this technique is unfortunate and readily remedied by its use in simple, but effective treatments.

5. Canines with mesial undercuts are ideal abutment teeth, but enameloplasty or addition of RBC can be used to quickly modify deficient teeth.

6. Long anterior rests are used on the mesial abutment teeth, and traditional rests, retentive elements and clasps are used on the posterior teeth.

7. Additional benefits may include psychological and periodontal advantages over traditional prostheses.

8. Patient education is important to ensure that the mesial undercuts are protected and that the patient and any other clinicians with whom s/he interacts can comfortably insert or remove the appliance.

\section{Conclusion}

In a world increasingly dominated by fixed implant restorations, the RPD is often regarded as the unaesthetic last resort for the patient who is not a candidate financially or physiologically for more popular restorative techniques.

This case study shows that not only can highly aesthetic results be achieved using rotational path of insertion RPDs, but that these cases are accessible to the modern clinician without significant investment in time or any new equipment.

It is the author's hope that adding this case to the modern literature serves to encourage others to explore and utilize this technique to benefit patients for whom this treatment is appropriate.

\section{Acknowledgments}

The author would like to thank the faculty of the University of Florida, College of Dentistry, Prosthodontics Department; in particular, Dr. Luis Rueda, for his encouragement to learn about and pursue this treatment modality.

\section{References}

1. Osada H, Shimpo H, Hayakawa T, Ohkubo C. Influence of thickness and undercut of thermoplastic resin clasps on retentive force. Dent Mater J. 2013;32(3):381-389. [Full Text Links] [Pubmed] Google Scholar(22) Scopus(8)

2. Tannous F, Steiner M, Shahin R, Kern M. Retentive forces and fatigue resistance of thermoplastic resin clasps. Dent Mater. 2012;28(3):273--278.

[Full Text Links] [Pubmed] Google Scholar(93) Scopus(44)

3. Alikhasi M, Monzavi A, Gramipanah F, et al. Rotational path removable partial denture: A literature review. J Indian Prosthodont Soc. 2007;7(3):143-146.

[Full Text Links] Google Scholar(2) Scopus(0)

4. Jacobson TE. Rotational path partial denture design: A 10year clinical follow-up--Part I. J Prosthet Dent. 1994;71(3):271277.

[Full Text Links] [Pubmed] Google Scholar(27)Scopus(15)

5. Jacobson TE. . Rotational path partial denture design: A 10year clinical follow-up--Part II. J Prosthet Dent. 1994;71(3):278282.

[Full Text Links] [Pubmed] Google Scholar(18) Scopus(9)

6. Wiener RC, Wiener MA, McNeil DW. Comorbid depression/ anxiety and teeth removed: behavioral risk factor surveillance system 2010. Community Dent Oral Epidemiol. 2015;43(5):433-443.

[Full Text Links] [Pubmed] Google Scholar(11) Scopus(4)

7. Donovan TE, Derbabian K, Kaneko L, Wright, R. Esthetic considerations in removable prosthodontics. J Esthet Restor Dent. 2001;13(4):241-53.

[Full Text Links] [Pubmed] Google Scholar(50) Scopus(18)

8. Jacobson TE, Krol AJ. Rotational path removable partial denture design. J Prosthet Dent. 1982;48(4):370-376. [Full Text Links] [Pubmed] Google Scholar(61) Scopus(41)

9. Goncalves LM, Bezerra-Junior JR, Benatti BB, Santana IL. Improving the esthetic replacement of missing anterior teeth: interaction between periodontics and a rotational path removable partial denture. Gen Dent. 2011;59(3):190 194.

[Full Text Links] [Pubmed] Google Scholar(5) Scopus(2)

10. Ivanhoe JR. Laboratory considerations in rotational path removable partial dentures. J Prosthet Dent. 2000;84(4):470472. [Full Text Links] [Pubmed] Google Scholar(8) Scopus(3) 
The author is a DMD Candidate at the University of Florida and also earned an MBA 'with distinction' from Cornell University. Prior to dental school matriculation, he founded and served as chairman and CEO of a biomedical robotics startup which produces tissue engineering research equipment. He also holds an issued and several pending US Patents and is licensed to practice patent prosecution before the US Patent and Trademark Office. He has published academic work in business management, biomedical additive manufacturing, and he is approaching publication of a study aid book for dental anatomy.

\section{Ouestions}

\section{What are the unique required components of the rRPD?}

口a. Long Anterior Rests;

b. Rigid Retainers;

ac. Mesial Anterior Undercuts;

$\square$ d. All of the Above.

\section{Where do the rigid retainers lock into the teeth to create retention?}

$\square$ a. Anterior Rests;

ab. Mesial Undercuts;

ac. Buccal Undercuts;

d. Conventional Posterior Clasps.

\section{What case type(s) is/are appropriate for using the method described in the case report?}

Da. Kennedy Class IV RPD;

ab. Kennedy Class I Mod I RPD;

ac. Kennedy Class II Mod I RPD;

$\square d$. All of the Above.

\section{How is surveying a rRPD different from a traditional RPD?}

$\square$ a. There is no difference;

b. Specialized surveyor must be purchased;

$\square c$. The cast must be surveyed in two planes;

$\square d$. Surveying is not necessary to fabricate an rRPD.

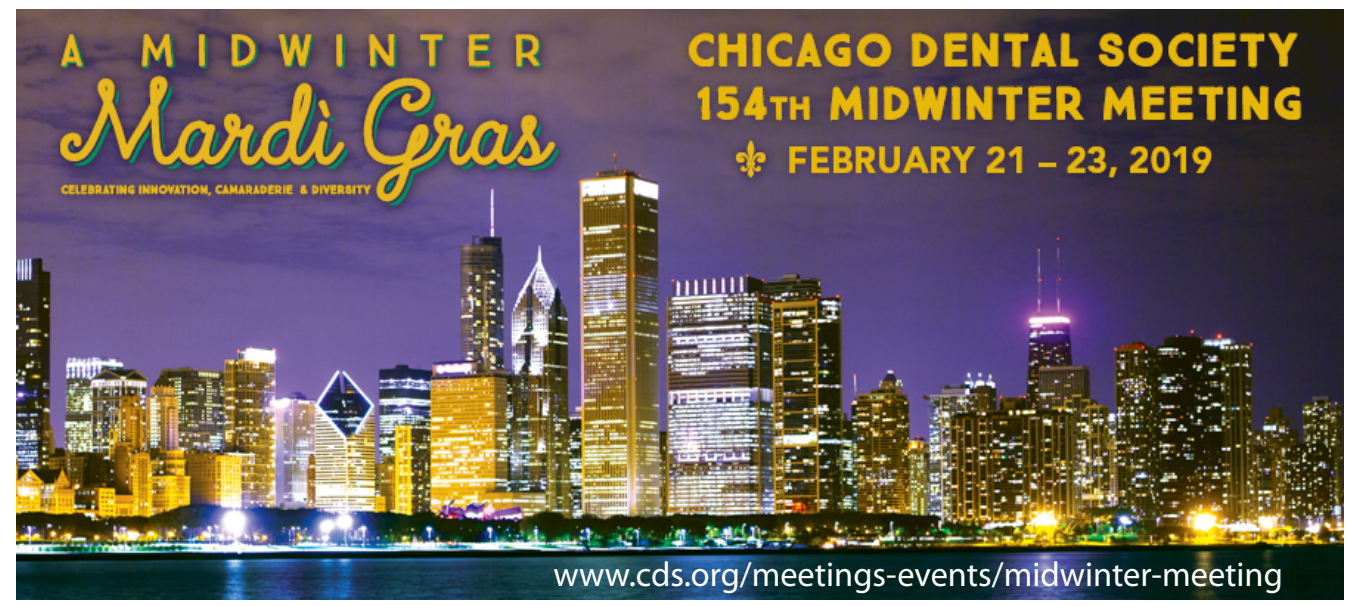

